\title{
Perspective
}

PERSPECTIVE Actualité en histoire de l'art

2| 2007

La Grande-Bretagne/Période moderne

\section{À la recherche de la formule perdue ? Les méthodes en histoire de l'architecture en question}

\section{Flaminia Bardati}

\section{Q OpenEdition}

1 Journals

Édition électronique

URL : http://journals.openedition.org/perspective/3852

DOI : 10.4000/perspective.3852

ISSN : 2269-7721

\section{Éditeur}

Institut national d'histoire de l'art

\section{Édition imprimée}

Date de publication : 30 juin 2007

Pagination : 395-400

ISSN : 1777-7852

\section{Référence électronique}

Flaminia Bardati, « À la recherche de la formule perdue ? Les méthodes en histoire de l'architecture en question », Perspective [En ligne], 2 | 2007, mis en ligne le 31 mars 2017, consulté le 01 octobre 2020. URL : http://journals.openedition.org/perspective/3852 ; DOI : https://doi.org/10.4000/perspective. 3852

Ce document a été généré automatiquement le 1 octobre 2020. 


\title{
À la recherche de la formule perdue ? Les méthodes en histoire de l'architecture en question
}

\author{
Flaminia Bardati
}

\section{RÉFÉRENCE}

Méthodes en histoire de l'architecture, $\mathrm{n}^{\circ}$ 9-10 des Cahiers de la recherche architecturale et urbaine, 2002. 240 p., 100 fig. n. et b. ISBN : 2-85822-659-8; $30 €$.

Nouvelles approches en Architecture, $n^{\circ} 59$ d'Histoire de l'art, 2006. 172 p., 75 fig. n. et b.

ISBN : 2-85056-960-7 ; $30 €$.

Dana Arnold, Elvan Altan Ergut, Belgin Turan Özkaya, Rethinking Architectural

Historiography, Londres/New York, Routledge, 2006. 251 p., 27 fig. n. et b. ISBN :

0-415-36085-4; 31,50 £.

Depuis quelques années, la réflexion sur les méthodes d'analyse dans le domaine de l'histoire de l'architecture semble reprendre vigueur. Parmi les nombreux ouvrages récents qui ont abordé ce sujet selon des traditions et des points de vues différents et complémentaires, trois recueils méritent de retenir l'attention.

2 Le numéro 9-10 des Cahiers de la recherche architecturale et urbaine, qui porte pour soustitre Méthodes en histoire de l'architecture, visait à comparer les diverses expériences des écoles européennes - française, italienne et allemande notamment - avec celles des écoles américaines, en abordant toutes les périodes et toutes les facettes de la discipline. Plus récemment, le numéro 59 de la revue Histoire de l'art intitulé Nouvelles approches en Architecture et faisant suite au débat du colloque international « Repenser les limites, l'architecture à travers l'espace, le temps et les disciplines » (Paris, INHA/ Society of Architectural Historians, août-septembre 2005) privilégie les résultats des recherches actuelles en proposant au lecteur une réflexion sur les méthodes. Des objectifs semblables sont à la base de Rethinking Architectural Historiography, où l'accent 
est mis sur les particularités disciplinaires de l'architecture et sur la tradition historiographique et culturelle occidentale, dans le but de présenter une série d'essais ouverts à des expériences « différentes ».

3 Pourquoi l'architecture attire-t-elle autant de réflexions? Longtemps partagée entre les méthodes de l'histoire de l'art et les interprétations des architectes-concepteurs, des ingénieurs et des archéologues, la discipline a cherché, au cours des $\mathrm{xIX}^{\mathrm{e}}$ et $\mathrm{xx}^{\mathrm{e}}$ siècles, à mettre au point ses propres catégories d'analyse. Par l'approche "culturelle ", qui unissait l'attention d'Henri Focillon pour la forme aux enseignements de Aby Warburg, Fritz Saxl et Erwin Panovsky comme dans le cas d'André Chastel, ou à travers une démarche scientifique, qui utilisait d'abord l'analyse documentaire et le relevé d'architecture comme dans le cas de Gustavo Giovannoni - pour ne citer que deux des maitres qui ont influencé des générations de chercheurs -, les historiens de l'art et les architectes-historiens ont cherché à définir les spécificités de l'histoire de l'architecture en tant que discipline autonome. Ce passage a été essentiel pour analyser avec un regard neuf les patrimoines architecturaux européens et a donné lieu à des monographies et des ouvrages de synthèses fondamentaux, comme, par exemple, les actes des colloques organisés au C.E.S.R. de Tours (collection "De Architectura »), le Bramante architetto d'Arnaldo Bruschi (Bari, 1969) ou la collection Storia dell'architettura italiana récemment publiée par Electa. Mais ces approches, qui ont révolutionné en leur temps la conception de l'histoire de l'architecture, requièrent une mise à jour face à l'évolution des méthodes et à la prise en compte de l'architecture du $\mathrm{xx}^{\mathrm{e}}$ siècle qui repose notamment sur des sources différentes, telles que les interviews des artistes, la documentation numérique ou la réaction des médias.

Ce besoin d'élargir les domaines d'étude et de faire évoluer les méthodes est dû, probablement, à la nature hybride de l'architecture et, par conséquent, à la nécessité de préciser sa place dans l'éventail des sciences humaines ${ }^{1}$. Certes, sa parenté avec l'histoire de l'art implique des similitudes de méthode mais d'autres disciplines telles l'archéologie, l'ingénierie, le dessin, la conservation, l'histoire sociale ou économique, l'histoire urbaine, pour ne citer que les plus proches, sont concernées. Une discipline riche et multiforme donc, dont les approches méthodologiques varient souvent selon les périodes considérées et les traditions académiques natio-nales. Si l'architecture antique reste étroite-ment liée à l'archéologie et jouit des avancées scientifiques de ce domaine, la période contemporaine oscille entre l'approche scientifique et l'influence de la critique qui ferait pencher pour une histoire militante de l'architecture, pour reprendre l'expression de Bruno Zevi. L'intérêt pour l'affinité entre architecture et philosophie apparaît dans l'anthologie de Neil Leach en 1997 (Rethinking Architecture. A reader in cultural theory, Londres/New York, 1997), où la compréhension des parcours de la création contemporaine est proposée à travers l'analyse de textes de Derrida, Habermas, Eco, Barthes, Vattimo, Lefebvre, Heidegger, Bloch ou Tschumi. Pour Leach, l'architecture a subi une sorte de métamorphose ou mieux, de glissement vers d'autres domaines : non seulement les architectes et les théoriciens de l'architecture deviennent plus réceptifs à la culture dans son sens le plus large, mais les théoriciens de la culture, les philosophes et les sociologues sont de plus en plus engagés dans les problèmes propres à l'architecture. Ces approches, qui concernent en principe la critique d'art et le projet, ont eu parfois une forte influence sur les méthodes d'analyse adoptées pour l'histoire de l'architecture contemporaine, bien que dans beaucoup d'écoles 
européennes l'approche philologique et la comparaison des sources figurées et textuelles restent la base méthodologique des recherches².

5 L'exploration des liens interdisciplinaires entre l'archéologie, la sociologie, l'anthropologie, l'histoire de la construction et des technologies, le recours aux instruments traditionnels de l'histoire de l'art tels que l'iconologie et l'étude des symboles, la philologie, l'approche stylistique, les parcours spécifiques de certaines institutions de recherche autres que les universités et, finalement, l'exploitation de nouveaux outils tels que les logiciels pour la restitution en trois dimensions, mais aussi les bases de données, la numérisation des images, la dendrochronologie, étaient les idées-forces des essais de la livraison de Méthodes... de 2002. Ce vaste panorama était enrichi par la présence de chercheurs relevant de l'ensemble de la culture occidentale, européenne et américaine. La recherche des spécificités de la discipline, la différentiation de catégories d'analyse " intérieures » et " extérieures " comme, par exemple, la notion de bourgeoisie utilisée pour le Moyen Âge, la mise en garde contre les risques de ces dernières font de ce recueil un point de départ essentiel pour le renouvellement méthodologique, devenu indispensable face à la multiplication des connaissances et des moyens de recherche.

6 Bien que, dès la préface, son approche se déclare différente - surtout avec la dénonciation des «Imperialistic Eurocentric ways in which architectural histories have been constructed» (D. Arnold, Rethinking architectural historiography, p. xvii) - Rethinking Architectural Historiography cherche aussi à établir des liens interdisciplinaires entre l'histoire de l'architecture et d'autres branches de l'histoire de la culture, passant de l'architecture turque à la relecture du traité de Filarete, de la réflexion sur les conditions de l'enseignement de l'histoire de l'architecture en Amérique du Nord à l'utilisation des modèles numérisés pour les restitutions et, en même temps, à des excursus théoriques et à des exemples de recherches. Les réflexions autour du problème de la redéfinition du domaine disciplinaire et la volonté de présenter une sorte de carte géographique et socioculturelle des positions historiographiques actuelles, à l'attention des chercheurs de toute origine, avec l'ambition de ne privilégier aucunes d'entre elles, sont particulièrement intéressantes (E. Altan Ergut, B. Turan Özkaya, Rethinking architectural historiography, p. 1).

7 L'objectif de dépasser les limites ou mieux, de redéfinir les axes géographiques et chronologiques du domaine de recherche de l'histoire de l'architecture caractérise aussi les Nouvelles approches... de 2006. La multiplicité des thèmes abordés, de la France du XIV siècle à la création des villes coloniales aux $\mathrm{XIX}^{\mathrm{e}}-\mathrm{XX}^{\mathrm{e}}$ siècles en passant par les sources de l'architecture postmoderne aux États-Unis vise à mettre en évidence à la fois les problématiques nouvelles et les méthodes pour les étudier. L'attention à ce qui est défini comme le «banal en architecture » pour reprendre le sous-titre - c'est-à-dire le tissu urbain, l'architecture autre que l'œuvre d'art signée par l'architecte de renom tient une place particulière, en prenant en compte les études (et les réévaluations) de Giovannoni, Loyer, Rivière et Cassan. On pourrait renvoyer aux méthodes de Saverio Muratori et Gianfranco Caniggia étudiées par Jean Castex, «La critique des types a-telle une influence sur l'histoire de l'architecture? (dans Méthodes..., p. 57-68).

8 À travers des points de vue très différents et sur la base de réflexions théoriques ou de résultats de recherches personnelles, ces trois ouvrages prennent en considération la quasi-totalité des facettes du problème de base: comment renouveler les études d'architecture et les champs d'enquête qu'elle recouvre, comment intégrer les 
nouvelles techniques dans une démarche disciplinaire sérieuse, comment ouvrir le débat aux domaines voisins ou complémentaires?

9 À côté de ces travaux, une nouvelle conscience de l'importance du rapprochement méthodologique entre histoire de l'architecture et histoire de l'art semble émerger d'une série de colloques, souvent organisés dans le cadre d'écoles doctorales et de conventions de cotutelle universitaires européennes, mais également du débat international proposé par plusieurs chercheurs américains ${ }^{3}$.

Dans ce cadre, Moyen Âge, Renaissance et époque moderne semblent garder une certaine spécificité disciplinaire, fondée dès la fin du xix $x^{e}$ siècle, sur les enseignements des maîtres des diverses écoles nationales. Toutefois, des paramètres nouveaux ont radicalement changé l'approche des chercheurs et leurs méthodes : pour se limiter à la célèbre triade vitruvienne, la structure des bâtiments (firmitas) est désormais étudiée grâce aux modèles d'analyse de l'archéologie et de l'ingénierie (à l'aide des restitutions virtuelles en trois dimensions). Pour la beauté (venustas), le concept de "style » s'est trouvé enrichi, sinon renouvelé, par l'étude des rapports et du transfert des formes entre centre et périphérie. Enfin, dans le domaine de la fonction (utilitas), l'étude des modes de vie, des usages sociaux et du cérémonial a révolutionné la compréhension des espaces intérieurs. Dans les trois ouvrages considérés, la Renaissance et l'époque moderne n'occupent malheureusement pas la place principale, étant donné que la réflexion sur la période contemporaine a davantage pris le dessus. Cependant, on dénote d'une part la volonté de systématiser les méthodes et les résultats de la recherche durant les trente dernières années, de l'autre, la nécessité d'exploiter de nouveaux domaines ou de réexaminer des sujets connus selon des points de vue et des paramètres inédits.

11 Quelques essais des Méthodes... de 2002 se réfèrent à la première tendance. La formation d'architecte-historien italien actuel renferme intrinsèquement, selon Paolo Fiore, les enseignements de Gustavo Giovannoni, Bruno Zevi, Guglielmo de Angelis d'Ossat, Manfredo Tafuri et Arnaldo Bruschi. Les méthodes de ces maîtres, parfois divergentes mais complémentaires, portent d'abord sur l'étude du contexte historique et urbain afin de comprendre les intérêts des commanditaires, puis sur la recherche d'archives et sur le relevé du bâtiment, considéré comme une étape essentielle pour la connaissance approfondie de l'architecture. Le recoupement de ces données et la confrontation avec d'autres édifices permettent de situer l'œuvre d'un point de vue stylistique et d'en évaluer la portée novatrice (P.F. Fiore, "Recherches en histoire et formation des architectes. L'exemple du palais della Rovere à Savone ", dans Méthodes..., p. 113-126). Côté français, la génération marquée par les enseignements d'André Chastel fait le point sur la révolution introduite par ce maitre. L'importance de la description scientifique du bâtiment et de son décor, l'attention nouvelle portée aux éléments constructifs et distributifs, au rôle du chantier, au rapport entre architecture et cérémonial, entre fonction et représentation, ont lentement mais radicalement changé l'approche de l'étude de la Renaissance française, ouvrant la voie à l'étude de l'architecture du XVII ${ }^{e}$ siècle et renouvelant les bases du dialogue franco-italien entre $\mathrm{XV}^{\mathrm{e}}$ et $\mathrm{XVII}^{\mathrm{e}}$ siècles (Jean Guillaume, "L'architecture de la Renaissance hier et aujourd'hui », dans Méthodes..., p. 53-56 ). Si l'énorme recensement du patrimoine 
architectural français effectué avec la création de l'Inventaire a permis d'avoir un regard approfondi et homogène sur la richesse nationale (Monique Chatenet, «L'aventure de l'Inventaire », dans Méthodes..., p. 133-136), la mise au point de grilles d'analyse valables dans les contextes urbains et ruraux et la publication du Vocabulaire de l'architecture ${ }^{4}$ ont permis à chercheurs et étudiants d'avoir une approche beaucoup plus scientifique de la discipline. Avec la même attitude, l'utilisation des outils des architectes tels que la cartographie permet de situer les monuments dans leur condition naturelle et d'évaluer les choix de projet selon des données objectives (F. Boudon, «Cartes sur table », dans Méthodes..., p. 69-80).

12 La réflexion sur l'emploi des nouveaux outils numériques concerne autant l'antiquité que la période moderne: en fait, si la restitution des projets non exécutés ou des édifices détruits constitue un axe essentiel de la recherche, car elle permet d'enrichir l'œuvre d'un architecte ou de récupérer les maillons perdus de la chaîne d'une typologie, la rigueur dans l'évaluation des hypothèses, essentielle pour une démarche scientifique, devient obligatoire lors de l'utilisation des logiciels qui amènent naturellement à l'exclusion de toutes les hypothèses impossibles du point de vue constructif (H. Schlimme, "Les restitution d'édifices en trois dimensions. Une façade non-réalisée pour Saint-Charles-aux-Quatre-Fontaines », dans Méthodes..., p. 109-112; D. Favro, "The digital disciplinary divide. Reactions to historical virtual reality models », dans Rethinking architectural historiography, p. 200-214).

13 Les essais des Nouvelles approches... et de Rethinking Architectural Historiography se réfèrent surtout à la seconde tendance, celle de l'allongement du questionnaire. Deux exemples de recherche proposés dans Nouvelles approches... montrent comment des sujets étudiés à maintes reprises peuvent être complètement transformés quand on aborde le thème de la résidence sous un jour nouveau. L'étude des édifices castraux de la fin du Moyen Âge a presque toujours considéré seulement les aspects fortificatifs. En prenant en compte les nécessités résidentielles - le chauffage et les latrines, et donc l'emplacement des cheminées et des conduites hydrauliques -, Christophe Moreau arrive à montrer l'évolution typologique et fonctionnelle de la forteresse au château moderne (C. Moreau, «Le confort dans les châteaux bourguignons aux $\mathrm{XIV}^{\mathrm{e}}$ et $\mathrm{xV}^{\mathrm{e}}$ siècles ", dans Nouvelles approches..., p. 17-28). Ce type de démarche, déjà utilisé pour l'époque moderne, apporte donc des nouveautés sensibles lorsqu'on l'applique à la fin du Moyen Âge. Mais la connexion entre la résidence et le mode de vie peut être encore exploitée en considérant des aspects quelque peu négligés. C'est le cas de la présence des femmes dans la vie parisienne de la première moitié du XviII ${ }^{e}$ siècle, qui amène Claire Ollagner à distinguer la spécificité du lieu de résidence féminin et à en dégager les caractéristiques par la comparaison des espaces destinés à l'homme et à la femme dans une même demeure et par l'analyse des hôtels commandés par des femmes seules (c. Ollagnier, "Architecture au féminin: le cas de l'hôtel parisien du $\mathrm{xvIII}^{\mathrm{e}}$ siècle (1700-1750) », dans Nouvelles approches..., p. 41-50).

Il convient donc d'examiner les sujets avec d'autres points de vue mais aussi de relire les documents en s'intéressant à des questions souvent négligées. Ainsi Sevil Enginsoy Ekici, se fondant sur les suggestions de Dominick LaCapra concernant l'interprétation des sources, propose une nouvelle lecture des passages que Filarete consacre au studiolo de Pierre de Côme de Médicis dans le palais de famille à Florence. Le rôle joué par la collection des manuscrits dans la mise en scène spatiale, destinée à la fois à être montrée aux hôtes importants et à la jouissance personnelle - intellectuelle, tactile et 
visuelle - de son propriétaire, peut être ainsi reconstruit (S. E. Ekici, « Reopening the question of document in architectural historiography. Reading (writing) Filarete's treatise on architecture for (in) Piero de' Medici's study ", dans Rethinking architectural historiography, p. 121-134).

Certaines thématiques très approfondies pour les $\mathrm{XIX}^{\mathrm{e}}-\mathrm{XX}^{\mathrm{e}}$ siècles se révèlent d'un grand intérêt quand on les transpose aux périodes précédentes. Ainsi le décor urbain devient un nouveau sujet d'étude lorsqu'il s'agit de considérer les promenades plantées et les jardins publics aux $\mathrm{xVII}^{\mathrm{e}}$-XvIII ${ }^{\mathrm{e}}$ siècles. Le rôle grandissant que ces lieux acquièrent montre l'évolution de la pensée architecturale sur l'image de la ville et de l'importance croissante de l'espace public (S. Pascalis, « La nature citadine : promenades plantées et jardins publics en France aux XVII et XVIII siècles ", dans Nouvelles approches..., p. 29-40). De même, les méthodes de l'archéologie peuvent donner des résultats très intéressants une fois appliquées à la période moderne. $\mathrm{Si}$, comme dans le cas de la récente monographie sur Maulnes, l'apport des archéologues a été décisif pour la compréhension et la datation de certaines structures du château ${ }^{5}$, l'étude de cas présentée par Roger $\mathrm{H}$. Leech concernant l'analyse de la Trinity Area de Frome (Sommerset), réalisée entre 1665 et 1725, montre comment les procédés d'analyse normalement appliqués sur des sites anciens se révèlent également valables dans le cas d'une implantation proto-industrielle (R. H. Leech, «Building archaeology. Context and points of convergence", dans Rethinking architectural historiography, p. 24-35). La photographie aérienne a permis de comprendre le tracé des rues, les processus de construction des bâtiments, la formation des îlots ainsi que la structure des parties démolies durant les années 1960. L'analyse directe du bâti, complétée par l'exploitation des sources textuelles, a mis en lumière la création des plans-types et leur distribution intérieure. La réalisation des nouveaux plans, à l'instar des reconstructions des sites anciens, a révélé la complexité des relations entre les habitations contiguës, en contribuant à la compréhension du processus d'implantation et de construction.

Certes, l'ensemble des ouvrages ci-dessus mentionnés n'apporte pas de "recette » à l'histoire de l'architecture et il est parfois nécessaire d'examiner certains procédés et leurs résultats avec un esprit fort critique. Toutefois, la volonté de renouveler les méthodes et de débattre des nouvelles attitudes possibles est l'indice d'un changement dans l'approche critique et scientifique de l'architecture. La contemporanéité de ces réflexions issues de diverses écoles européennes, américaines et medio-orientales, dénote le besoin d'élargir le débat disciplinaire et de l'ouvrir à d'autres secteurs scientifiques ainsi qu'à des chercheurs venus de tous les horizons.

\section{NOTES}

1. Pour ne citer que deux exemples assez éloignés l'un de l'autre, pour John Pinto elle pourrait être classée parmi les sciences humaines, pour Antoine Picon elle se rapprocherait plutôt de l'ingénierie (John Pinto, «L'histoire de l'architecture, une discipline des sciences humaines. Les enjeux du rapprochement entre historiens de l'art et architectes », dans Méthodes..., p. 161-168; Antoine Picon, "Architecture, sciences et techniques. Problématiques et méthodes", dans 
Méthodes..., p. 151-160). Selon les différents points de vue, elle est considérée comme faisant partie des arts visuels ou étroitement liée à la sociologie, comme le démontrent les approches didactiques différentes entre facultés d'histoire de l'art et écoles d'architecture.

2. Claudia Conforti, «Instruments et méthodes d'études de l'architecture contemporaine. Un parallèle avec l'histoire de l'architecture de la période moderne », dans Méthodes..., p. 127-132, insiste sur ce point. En même temps, plusieurs essais mettent en évidence l'existence de sources nouvelles et spécifiques pour l'architecture contemporaine, en relation à la fois avec les techniques et avec les nouveaux thèmes de projet, qui ont introduit des paramètres inédits. Dans cette optique, l'intérêt pour l'architecture des villes coloniales des Nouvelles Approches... trouve son équivalent dans celui pour les réalisations de la Turquie dans Rethinking Architectural Historiography.

3. Voir, à titre d'exemple, Alina A. Payne, "Architectural History and the History of Art ", dans Journal of the Society of Architectural Historians, 58-3, septembre 1999, p. 292-300, et Kathryn Fischer Taylor, « Architecture's place in Art History: Art or Adjunct? », dans The Art Bulletin, LXXXIII-2, juin 2001, p. 346.

4. Sous la direction de Jean-Marie Pérouse de Montclos, Paris, 1972.

5. Monique Chatenet, Maulnes. Archéologie d'un château de la Renaissance, Paris, 2004.

\section{INDEX}

Mots-clés : histoire de l'architecture, pluridisciplinarité, architecte-historien, patrimonialisation, études, méthodologie, discipline, historiograhie

Keywords : architecture history, multidisciplinary approach, architect-historian, heritage, studies, methodology, historiography

Index géographique : France, Allemagne, Angleterre, Italie, Europe

Index chronologique : 1900, 2000

\section{AUTEURS}

\section{FLAMINIA BARDATI}

EPHE / Università della Tuscia (Viterbe) 